\title{
An unusual case of intrathoracic Castleman's Disease
}

\author{
Rachel Schoon BSc', Liam Mulroy MD², Zhaolin Xu MD³, Drew Bethune MD MSc \\ ${ }^{1}$ Class of 2016, Faculty of Medicine, Dalhousie University \\ ${ }^{2}$ Department of Radiation Oncology, Faculty of Medicine, Dalhousie University \\ ${ }^{3}$ Department of Pathology and Laboratory Medicine, Faculty of Medicine, Dalhousie University \\ ${ }^{4}$ Division of Thoracic Surgery, Faculty of Medicine, Dalhousie University
}

\begin{abstract}
Castleman's disease (CD) is a rare, non-clonal disease of the lymph nodes that may develop at a single site (unicentric form) or multiple sites (multicentric form) throughout the body. Histopathologically, CD is differentiated into a hyaline vascular variant, a plasma cell variant and a mixed cell type. Classically, unicentric CD is of the hyaline vascular variety while multicentric disease is of the plasma cell or mixed cellularity forms. We report an asymptomatic patient whose imaging revealed isolated left hilar lymphadenopathy. After thoracotomy, lymph node excision biopsy was performed, and histopathological examination revealed plasma cell histology. Unicentric plasma cell presentation is a rarely seen variant of CD.
\end{abstract}

A 64-year-old female, with no reported history of Acardiovascular disease or previous symptoms, presented to her primary care provider with left-sided chest pain. As part of a standard chest pain workup to rule out cardiac and non-cardiac causes, a posterioranterior chest X-ray (Figure 1) was performed and revealed a left hilar mass, initially thought to be related to the pulmonary artery. Assessment for cardiovascular disease was found to be negative and attention was then focused on the left hilar mass.

Past medical history revealed minimal smoking history (cessation over 25 years ago, less than five pack year history), with good exercise tolerance and no ongoing chest symptoms. She experienced an episode of pneumonia several years earlier, and was informed there was some residual "scarring" in her lung. Older chest X-rays were not available for review. The patient was not experiencing any B symptoms, and reported that her appetite and energy levels were good.

A chest computerized tomography (CT) scan was ordered and showed a solitary soft tissue mass lying between the left pulmonary artery and the descending aorta measuring $4 \mathrm{~cm}$ in diameter (Figure 2). The mass was irregularly shaped and found to abut the left main stem bronchus medially with some superior extension toward the tracheobronchial angle. There was no evidence of necrosis or metastatic process noted on imaging.

A whole body positron emission tomography (PET) scan (Figure 3a and 3b) showed 18F-fluorosdeoxyglucose (FDG) uptake in the left hilar region, confirming the presence of a metabolically active primary lung mass.

\section{Surgical Interventions}

The patient was referred to a thoracic surgeon who recommended biopsy and excision. Bronchoscopy was unremarkable and a left video assisted thoracoscopic surgery (VATS) wedge resection was attempted. Shortly after the procedure was started it became evident that visualization was inadequate and the procedure was converted to a left posterolateral thoracotomy. This revealed a very large lymph node mass wrapped around the pulmonary artery at the hilum of the lung. A frozen section was taken but pathology was indeterminate. Extensive dissection around the mass was performed, but it was determined to be unresectable. Multiple biopsies were taken and felt to be compatible with a hematologic malignancy. The patient recovered well post-operatively.

\section{Pathology Report}

The surgical biopsies showed lymph nodes with significant expansion of the interfollicular zone by a polytypic population of plasma cells, as well as some lymphocytes and histocytes. Some fibrosis was present both within the lymph nodes and at the periphery. Vessels within the nodes appeared normal with no hyalinization. The initial suspicion of a hematological malignancy was ruled out as there was no evidence of clonal proliferation of Blymphocytes found in the lymph node sample. In consultation with hematopathology, a diagnosis of atypical lymphoplasmacytic infiltration favouring plasma cell type Castleman's Disease (CD) was made.

\section{Background}

$\mathrm{CD}$ is a rare form of lymph node hyperplasia that results in non-neoplastic lymphadenopathy. ${ }^{1}$ The disease was first described in 1956 and since that time reported cases have been infrequent. ${ }^{2}$ There are few comprehensive studies or reliable estimates of its incidence in the population. ${ }^{1,3-5}$ 


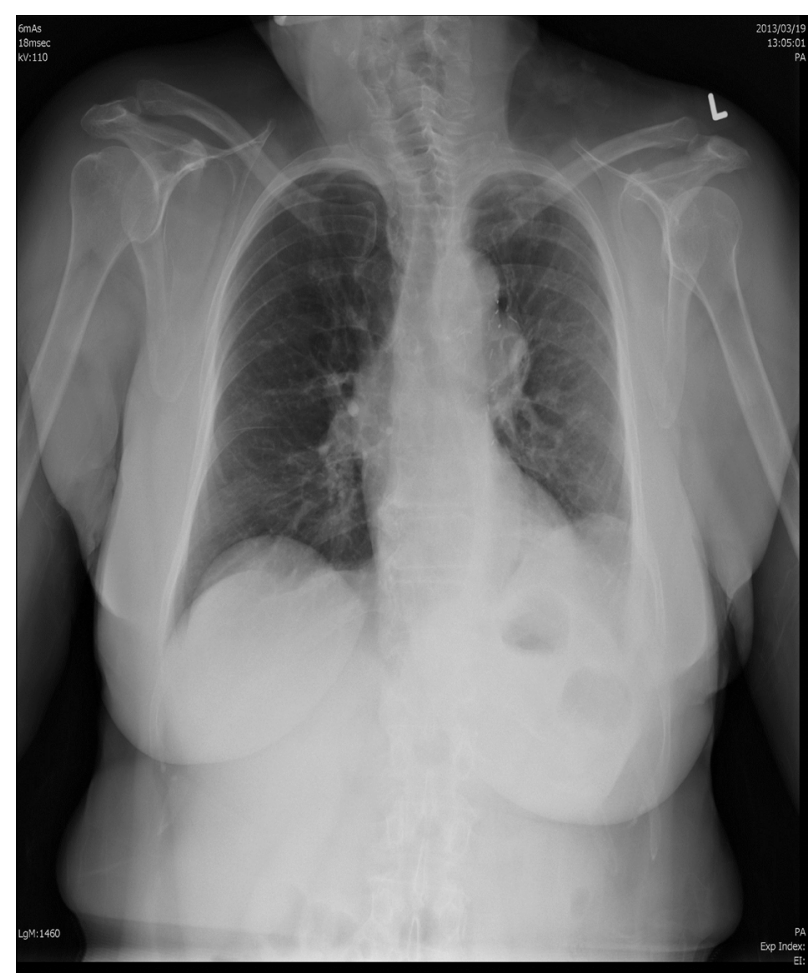

Figure 1. Posterior-anterior chest radiograph demonstrating a left hilar mass.

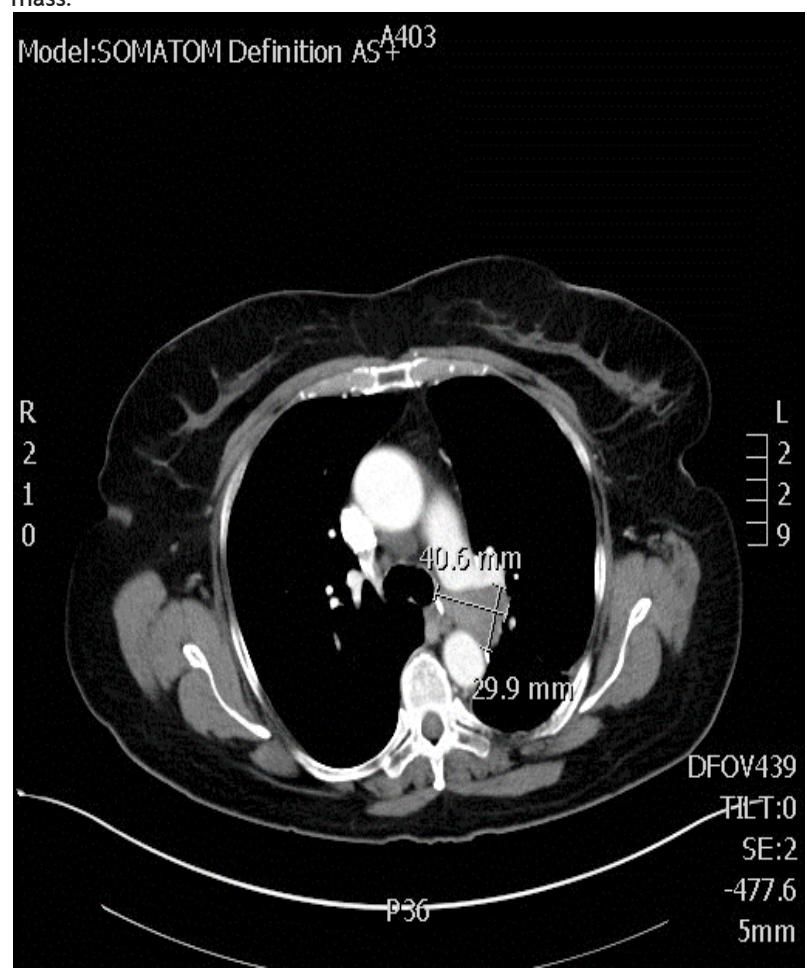

Figure 2. Transverse computed tomography image showing a mass between the descending aorta and the left pulmonary artery.

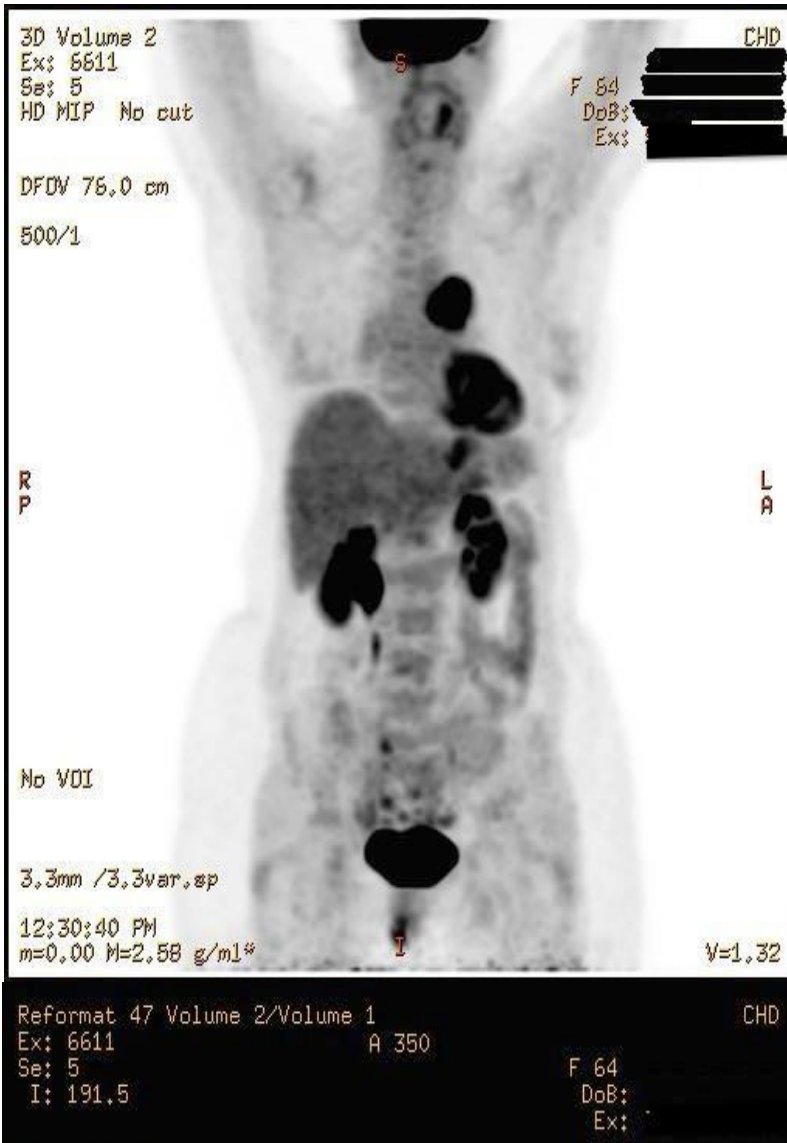

DFOV $70.0 \mathrm{~cm}$

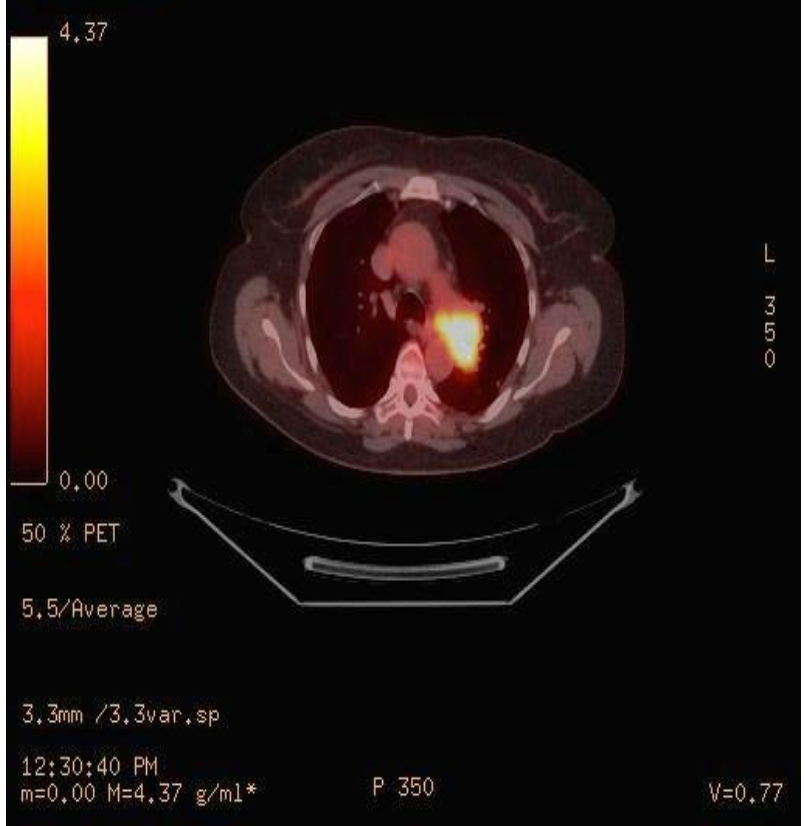

Figure 3. PET scan images showing FDG uptake in the left hilar area with normal physiological uptake in the bladder, kidneys, brain, and heart. A) Transverse view B) Coronal section 

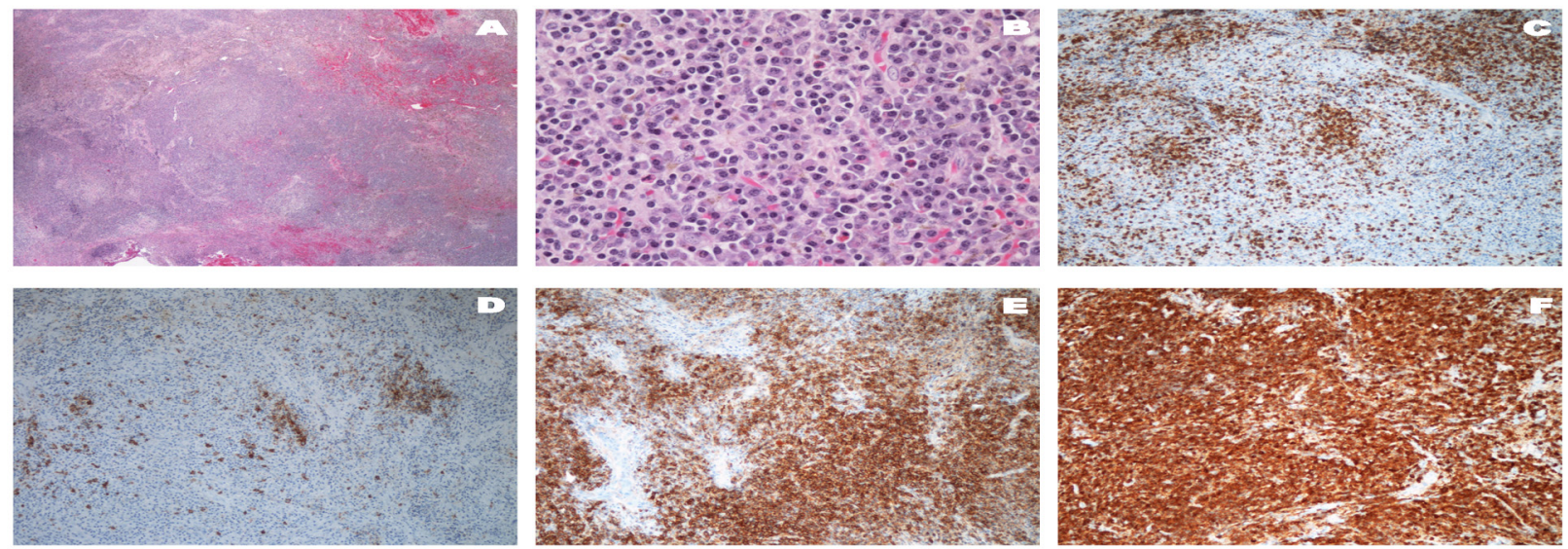

Figure 4: Histological stains demonstrating marked plasmacytosis of the interfollicular zone of the lymph node, characteristic of plasma cell Castleman's Disease. A) HE slide low power view (x20) B) HE slide high power view (x400) C) CD3 - T cell marker D) CD20 - B cell marker E) CD138 - plasma cell marker F) CD79a - positive for B cells and plasma cells. There is no light chain restriction for Kappa and Lambda in this case (slides not shown).

Clinically, two identified forms of the disease have been described, the indolent unicentric form, which presents as a solitary mass, and the more aggressive multicentric form that is characterized by widespread lymphadenopathy and symptoms including severe fatigue, night sweats, fever, weight loss, and anorexia., ${ }^{1,4-6}$ Histologically, the disease can be described as hyalinevascular type, plasma cell or mixed cell type. ${ }^{7}$ The hyaline-vascular form, characterized by small hyalinevascular follicles and capillary proliferation, is most common representing $85-90 \%$ of cases. ${ }^{3,8}$ The plasma cell form, characterized by large lymphoid follicles separated by sheets of plasma cells, and the mixed form account for the other $10-15 \%$ of cases.$^{3,8} \mathrm{~A}$ strong association has been shown to exist between the clinical and the histological classification of the disease (Table 1). Unicentric Castleman's Disease (UCD), is almost always of the hyaline vascular variety while multicentric Castleman's Disease (MCD) is almost always of the plasma cell type. ${ }^{3,4,6}$ The etiology of $\mathrm{CD}$ is unknown; however, new research suggests pathogenesis may be attributable to viral or human interleukin-6 (IL-6). ${ }^{9-11}$ IL-6 is a potent stimulator of B-cell proliferation

\begin{tabular}{lcc}
\hline & Unicentric & Multi-centric \\
\hline Clinical Findings & Solitary mass & $\begin{array}{c}\text { Wide-spread } \\
\text { lymphadenopathy }\end{array}$
\end{tabular}

\begin{tabular}{lcc}
$\begin{array}{l}\text { Predominant } \\
\text { pathology }\end{array}$ & Hyaline vascular & Plasma cell \\
$\begin{array}{l}\text { Systemic } \\
\text { symptoms }\end{array}$ & Rare & Common \\
Treatment & Surgical excision & Systemic therapy \\
\hline
\end{tabular}

Table 1. Differentiating features of unicentric and multicentric Castleman's disease. and is associated with hematologic malignancies such as multiple myeloma and lymphoma. ${ }^{1,10,12,13}$ An endogenous pyrogen, IL-6 induces the production of acute phase reactants by hepatocytes, resulting in the constitutional symptoms that characterize MCD as well as other lymphoid malignancies..$^{9,14}$ It also manufactures proteins that negatively interfere with the absorption and use of iron, resulting in iron deficiency anemia. ${ }^{9}$ Studies have demonstrated the overproduction of IL-6 by the germinal centers of hyperplastic lymph nodes in patients diagnosed with both UCD and MCD, with serum levels positively correlated with the severity of clinical symptoms. ${ }^{9-11,14}$

A viral form of IL- 6 has also been implicated in the pathogenesis of the disease. Human Herpes Virus-8 (HHV-8) is a human lymphotropic virus present in a high number of MCD cases that is especially prevalent in patients co-infected with HIV. ${ }^{9}$ The virus, also known as Kaposi's sarcoma-associated herpes virus, is present in almost all cases of MCD occurring in HIV-infected patients, as well as in $40-50 \%$ of HIV-negative MCD patients. ${ }^{1,15,16}$ Viral IL-6 (vIL-6) is structurally homologous to human IL-6 allowing it to bind to IL-6 receptors activating the downstream pathways leading to CD manifestations. ${ }^{9}$ As with human IL-6, a correlation exists between the quantity of HHV-8 in $C D$ affected tissues and the intensity of symptoms experienced with MCD. ${ }^{1,13}$

\section{Discussion}

Through careful history taking and in-depth medical investigations, our asymptomatic patient was diagnosed with unicentric-plasma cell variant (U-PCV) CD. A literature review found that U-PCV CD is an 
uncommon form of the disease that makes up less than $20 \%$ of all CD cases. ${ }^{8,10,17}$ It is characterized by the hypertrophy of a single lymph node or lymph node chain, and abnormal nodes are most frequently found in the abdomen..$^{10}$ Involvement of thoracic or mediastinal lymph nodes, as seen in our patient, is less common. ${ }^{10,17}$ Similar to the unicentric hyaline vascular variant (U-HVV), this form of the disease occurs equally in both genders, and is usually reported among patients in their third and fourth decades of life. ${ }^{10,15,17}$ Unlike $\mathrm{U}-\mathrm{HVV}$, the majority of U-PCV patients present with the constitutional symptoms and abnormal laboratory findings often associated with MCD., ${ }^{4,8,10,17}$ It has been suggested that some of the U-PCV patients reported in the literature may represent cases of misdiagnosed MCD, due to inadequate staging or low sensitivity of the applied tests. ${ }^{17}$ This seems unlikely in our patient as CT scans with contrast, a full body PET-CT scan and continued radiologic monitoring have not revealed any further lymph node involvement.

Prognosis for patients with U-HVV CD is generally good. Surgical excision is usually curative and radiotherapy can be used to treat partial resections. ${ }^{5,8}$ On the other hand, due to its extensive and aggressive nature, the prognosis for MCD is poor. ${ }^{5}$ Treatment may involve corticosteroids, anti-cancer drug therapy, antiviral medications, or antiparasitic drugs that inhibit the proliferation of lymphoid cells.,3,7,18 Targeting the IL-6 signalling cascade is a promising treatment strategy for patients with MCD. Clinical trials of monoclonal antibodies against IL-6 (siltuximab) and the IL-6 receptor (tocilizumab) have shown efficacy in reducing constitutional symptoms. ${ }^{8,9,12,15}$ More research is required, as clinical trial exclusion criteria includes co-infection with a serious illness, such as HIV/AIDS. ${ }^{12}$ Thus the benefit offered by these drugs is currently restricted to HIV/HVV-8 negative patients. ${ }^{9,12}$ MCD has been linked to a number of other illnesses including autoimmune hemolytic anemia, paraneoplastic pemphigus and POEMS syndrome. ${ }^{15,18}$ It also has a recognized potential to progress into malignant lymphoma. ${ }^{18}$ Long-term follow up is required to monitor for malignant changes in all cases of CD. With such a low incidence of confirmed unicentric-plasma cell cases, ${ }^{10,17}$ prognosis for this variant is not clear. Consequently, long-term outcomes for our patient are uncertain and the malignant potential of plasma cell CD must be kept in mind. However, based on her current condition and the incidental discovery of her disease, this outcome was not felt to be an immediate concern. After surgery the patient appeared well, and was referred to a radiation oncologist to discuss potential treatment options. As the mass was stable on a follow up CT scan performed approximately 6 months after surgery, radiation therapy and chemotherapy were not recommended. These modalities could be used in the future if there is local progression. Instead, the patient is being followed with regular imaging and clinical assessment. No changes have been detected after approximately two years of follow up

\section{References}

1. Roca B. Castleman's disease: a review. AIDS Reviews 2009;11(1):3-7.

2. Castleman B, Iverson L, Menendez VP. Localized mediastinal lymphnode hyperplasia resembling thymoma. Cancer 1956;9(4):82230

3. Zhou N, Huang CW, Liao W. The characterization and management of Castleman's disease. J Int Med Res 2012;40(4):1580-8.

4. Cronin DM, Warnke RA. Castleman disease: an update on classification and the spectrum of associated lesions. Adv Anat Pathol 2009;16(4):236-46.

5. Sarrot-Reynauld F. "Castleman's disease." Aug 2001. <https://www. orpha.net/data/patho/Pro/en/Castleman-FRenPro3618.pdf> (15 Aug 2015).

6. International Castleman's Disease Organization. "About Castleman's disease". $2010<$ http://www.castlemans.org/about> (June 2014).

7. Bonekamp D, Horton KM, Hruban RH, Fishman EK. Castleman disease: the great mimic. Radiographics 2011;31(6):1793-807.

8. Saeed-Abdul-Rahman I, Al-Amri AM. Castleman disease. Korean J Hematol 2012;47(3):163-77.

9. El-Osta HE, Kurzrock R. Castleman's disease: from basic mechanisms to molecular therapeutics. Oncologist 2011;16(4):497-511.

10. Casper $C$. The aetiology and management of Castleman disease at 50 years: translating pathophysiology to patient care. Br J Haematol 2005;129(1):3-17.

11. Nishimoto N, Sasai M, Shima Y, Nakagawa M, Matsumoto T, Shirai $\mathrm{T}$, et al. Improvement in Castleman's disease by humanized antiinterleukin-6 receptor antibody therapy. Blood 2000;95(1):56-61.

12. van Rhee F, Fayad L, Voorhees P, Furman R, Lonial S, Borghaei H, et al. Siltuximab, a novel anti-interleukin-6 monoclonal antibody, for Castleman's disease. J Clin Oncol 2010;28(23):3701-8

13. Waterston A, Bower M. Fifty years of multicentric Castleman's disease. Acta Oncologica 2004;43(8):698-704.

14. Yoshizaki K, Matsuda T, Nishimoto N, Kuritani T, Taeho T, Aozasa $\mathrm{K}$, et al. Pathogeneic significance of interleukin-6 (IL-6/BSF-2) in Castleman's disease. Blood 1989;74(4):1360-7

15. Dispenzieri A, Gertz MA. Treatment of Castleman's disease. Curr Treat Options Oncol 2005;6(3):255-66.

16. Oksenhendler E, Carcelain G, Aoki Y, Boulanger E, Maillard A, Clauvel J, et al. High levels of human herpesvirus 8 viral load, human interleukin-6, interleukin-10, and $C$ reactive protein correlate with exacerbation of multicentric Castleman disease in HIV-infected patients. Blood 2000;96:2069-73.

17. Miranda R, Khoury J. Unicentric Castleman disease. Atlas of Lymph Node Pathology New York: Springer; 2013.

18. Nistor C, Davidescu M, Ciuche A, Bluoss C, Motas N, Motas C, et al. A rare case of unicentric plasma cell type Castleman's disease in the mediastinum. Pneumologia 2010;59(1):32-5. 